\title{
THE ROLE OF PET/CT FOR DIFFERENTIAL DIAGNOSIS OF PULMONARY TUBERCULOSIS AND LUNG CANCER: CASE REPORT
}

\author{
Chaushev B., P. Bochev, ${ }^{1}$ I. Krasnaliev, A. Klisarova, ${ }^{2} \mathrm{Z}$. Georgieva, K. Yordanov, \\ J. Dancheva, C. Yordanova
}

Department of Nuclear Medicine, ${ }^{1}$ Department of Pathology, ${ }^{2}$ Department of Internal Medicine MBAL "St. Marina", Varna, Bulgaria

Reviewed by: assoc. prof. B. Balev

\begin{abstract}
Most solitary pulmonary nodules are discovered incidentally on chest radiographs and practically all patients undergo further diagnostic evaluation, since malignancy cannot be ruled out. Solitary pulmonary nodules are also frequently encountered in patients infected with Mycobacterium tuberculosis. Tuberculosis and lung cancer are very common diseases of the lungs. Subject: of our study was a sixty-one year old male who had complained of weakness and hemoptysis for two months. METHODS: The patient underwent aCTscan of the thorax with intravenous contrast, sputum examination, bronchoscopy with brush biopsy, tuberculin skin test (Mantoux), PET/CT scan of the whole body with 18 F- FDG. RESULTS: The PET/CT scan of thewhole body revealed a nodular lesion in the upper lobe of right lung with central zone of necrosis and irregular borders, increased uptake of 18F-FDG, SUV 5.8 in them and nodule lesion in the right suprarenal gland with high glucose metabolism in the borders SUV 4.3. CONCLUSION: Integrated PET/CT is better imaging technique than CT alone, PET alone and visual correlation of PET and CT.
\end{abstract}

Key words: PET/CT, 18F-FDG, nodular lesion, lung cancer

\section{INTRODUCTION}

Most solitary pulmonary nodules are discovered incidentally on chest radiographs and practically all patients undergo further diagnostic evaluation, since malignancy cannot be ruled out (10).

Survival in bronchial carcinoma is closely related to the stage of the disease at the time of diagnosis and a solitary pulmonary nodul represents a potentially curable stage amenable to surgery.

Solitary pulmonary nodules are also frequently encountered in patients infected with Mycobacterium tuberculosis. Tuberculosis and lung cancer are very common diseases of the lungs. The symptoms of these two types of diseases are very similar at the time of diagnosis.

The presence of cavitation is associated with a greater degree of infectiousness, likely due to higher organism burden (9).

Although the morphological findings of cavitation in patients with post primary tuberculosis are hardly distinguishable from malignant cavitary lesions, the presence of adja-

Address for correspondence:

B. Chaushev, Dept. of Nuclear Medicine and Radiotherapy

MBAL St.Marina, Varna

e-mail: bchaushev@gmail.com cent tree in bud lesions (5) or satellite nodules may help in differentiating tuberculosis from malignancy.

Cavitation in primary lung cancer is not rare. Cavitation detected on plain chest radiographs has been reported in $2 \%$ to $16 \%$ of primary lung cancers, $(1,2,3,4,6,7)$ and it is detected with computed tomography in $22 \%$ of primary lung cancer (8).

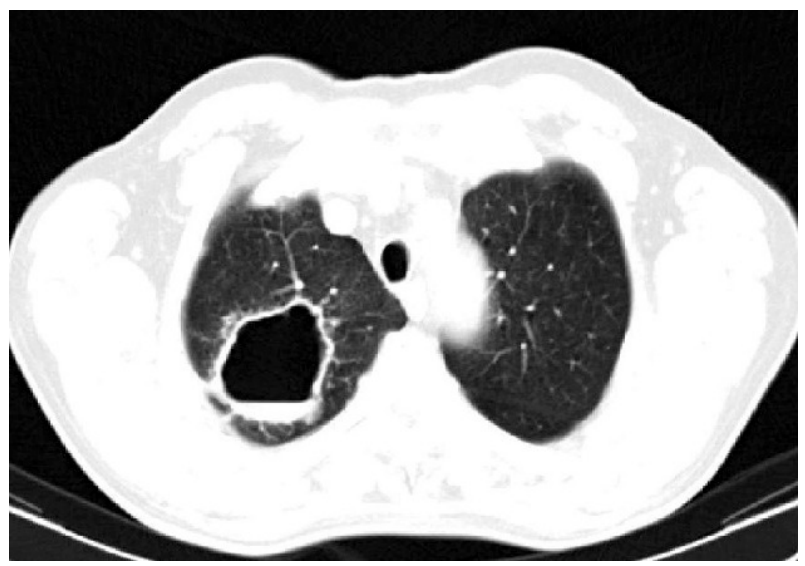

Fig. 1. CT scan of the thorax displays a cavern in the upper lobe of the right lung with pneumofibrotic borders

SUBJECT of our study was a sixty-one year old male who had complained of weakness and hemoptysis for two 


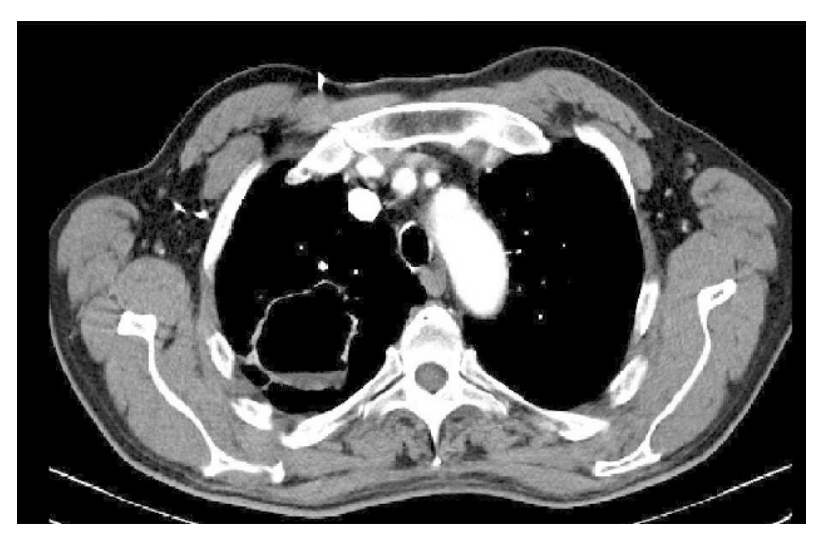

Fig. 2. CT scan of the thorax displays a cavern in the upper lobe of the right lung with air fluid level

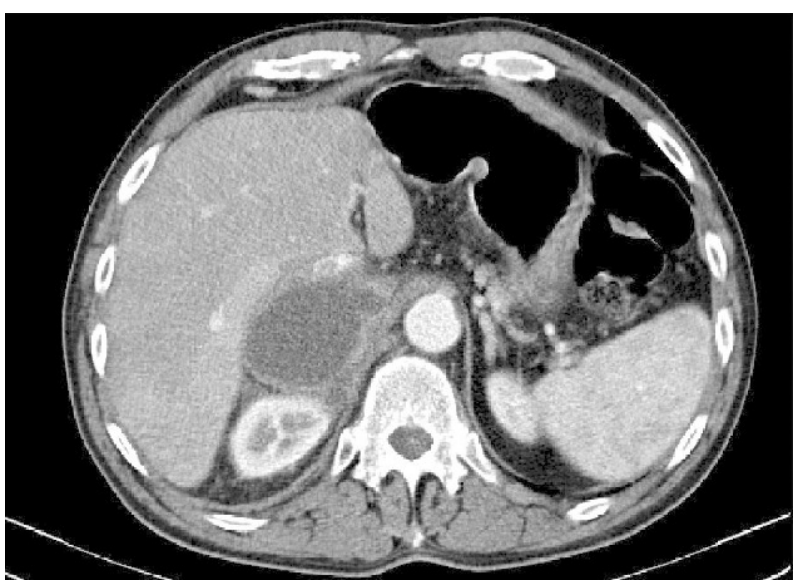

Fig. 4. CT scan of the thorax and upper part of abdomen displays hypodense tumor mass of the right suprarenal gland

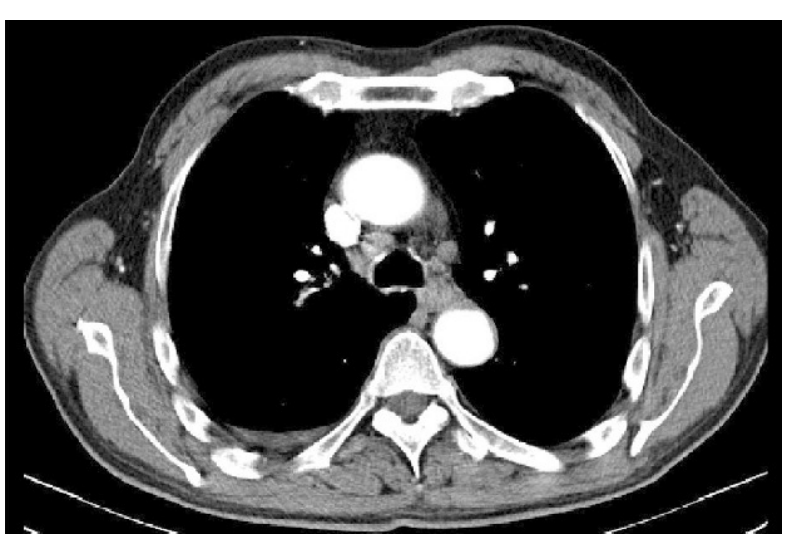

Fig. 3. CT scan of the thorax displays pleural effusion in the right pulmonary base

months. Physical exams: reduction in vesicular breath sounds in right and pain at the right upper part of abdomen after palpation.

\section{METHODS}

The patient underwent a CT scan of the thorax with intravenous contrast, sputum examination, bronchoscopy with brush biopsy, tuberculin skin test (Mantoux), PET/CT scan of the whole body with 18 F- FDG.

An 18 F-FDG PET/CT image was done with a PET/CT scanner (PHILIPS Gemini TF), consisting of dedicated lutetium orthosilicate full ring PET scanner and 16 slice CT scanner.
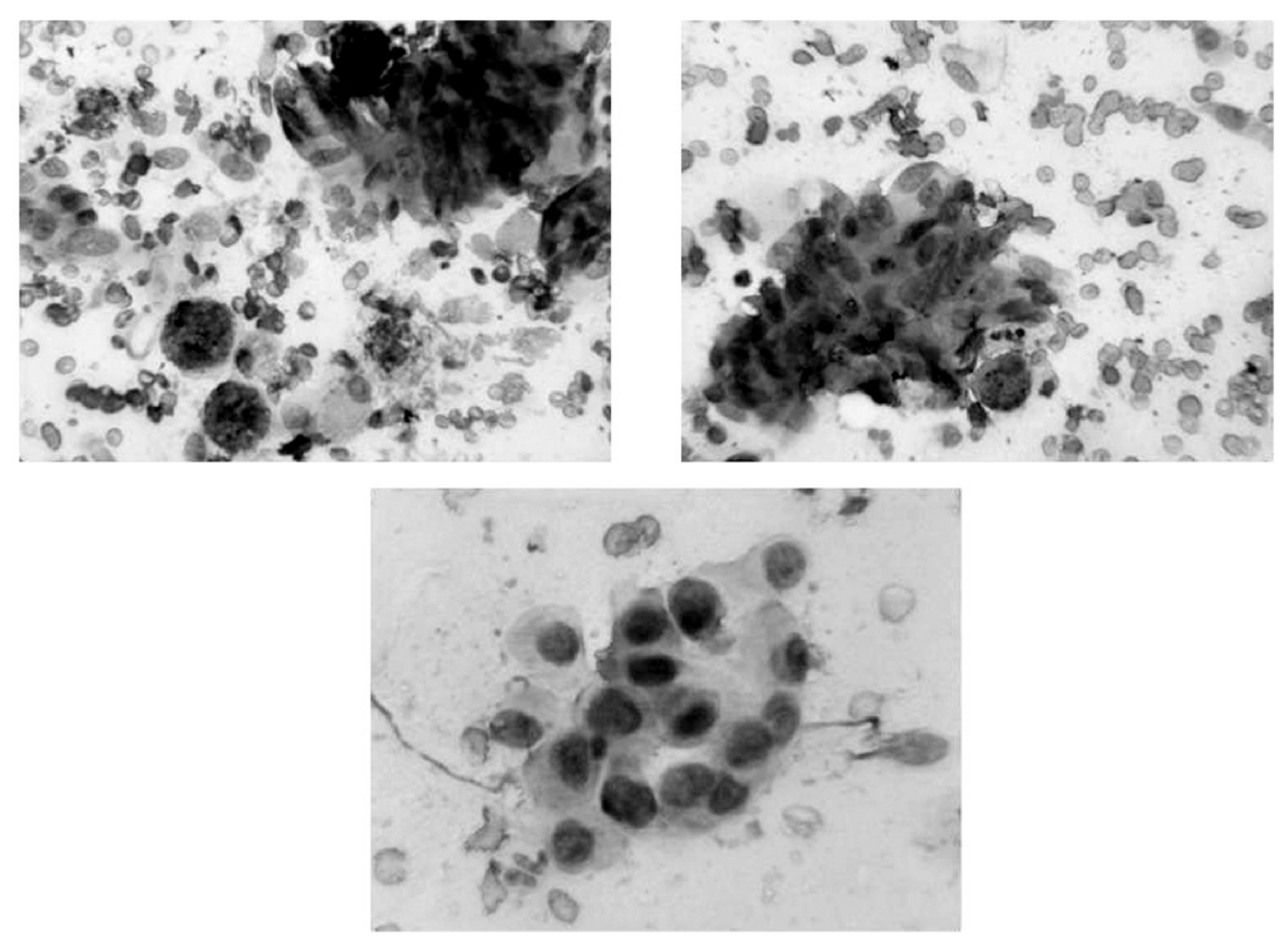

Fig. 5. Brush cytology-lung-Giemsa stain, 10x40 


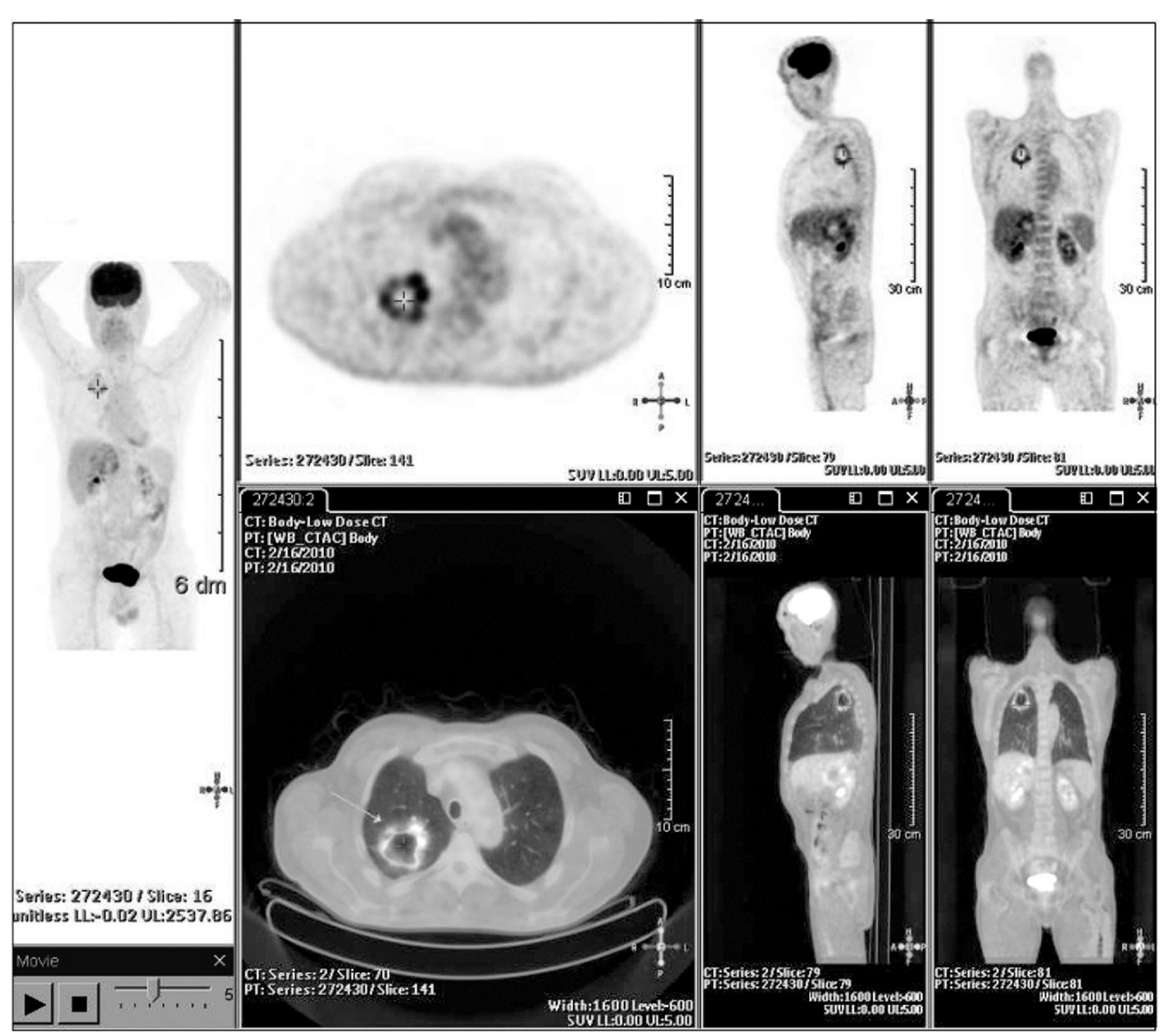

Fig. 6. PET/CT scan of the whole body displays a nodular lesion in the upper lobe of right lung with central zone of necrosis and irregular borders, increased uptake of $18 F-F D G$, SUV 5.8 in them

Standard patient preparation included at least 6 hours fasting and a serum glucose level of less than $120 \mathrm{mg} / \mathrm{dL}$ before $18 \mathrm{~F}-\mathrm{FDG}$ administration. PET/CT imaging was performed 60 minute after intravenous injection of $370 \mathrm{MBq}$ $(10 \mathrm{mCi})$ of $18 \mathrm{~F}-\mathrm{FDG}$.

At 60 minute after administration of $18 \mathrm{~F}-\mathrm{FDG}$, low dose CT $(50 \mathrm{mAs}, 120 \mathrm{kV})$ covering area from the scull to the proximal thighs was performed for the purpose of attenuation correction and precise anatomical localization. There after, emission scan was conducted in the three-dimensional mode. Emission scan time per mm was $39 \mathrm{~mm} / \mathrm{sec}$. PET data were obtained using a high-resolution whole-body scanner with an axial field of view of $57.6 \mathrm{~cm}$. The average total PET/CT examination time was 20 minutes.

After scatter and decay correction, PET data were reconstructed iteratively with attenuation correction and reoriented in axial, sagittal, and coronal slices. The row action maximum-likelihood algorithm was used for three dimensional reconstruction.

\section{RESULTS}

The CT scan of the thorax revealed a cavern in the upper lobe of the right lung with pneumofibrotic borders (Fig.1), air fluid level (Fig.2), pleural effusion (Fig.3) and hypodense tumor mass of the right suprarenal gland (Fig.4).

The bronchoscopy with the brush biopsy revealed inflammatory changes, pigmented macrophages, reactive and hyperplastic bronchial epithelium squamous metaplasia.

The sputum examination demonstrated resident microflora and the tuberculin skin test was negative.

The PET/CT scan of the whole body revealed a nodular lesion in the upper lobe of right lung with central zone of necrosis and irregular borders, increased uptake of 18F-FDG, SUV 5.8 in them (Fig.6) and nodule lesion in the right suprarenal gland with high glucose metabolism in the borders SUV 4.3 (Fig.7).

After the PET/CT scan of the whole body, fine needle aspiration biopsy was performed of the right suprarenal gland 


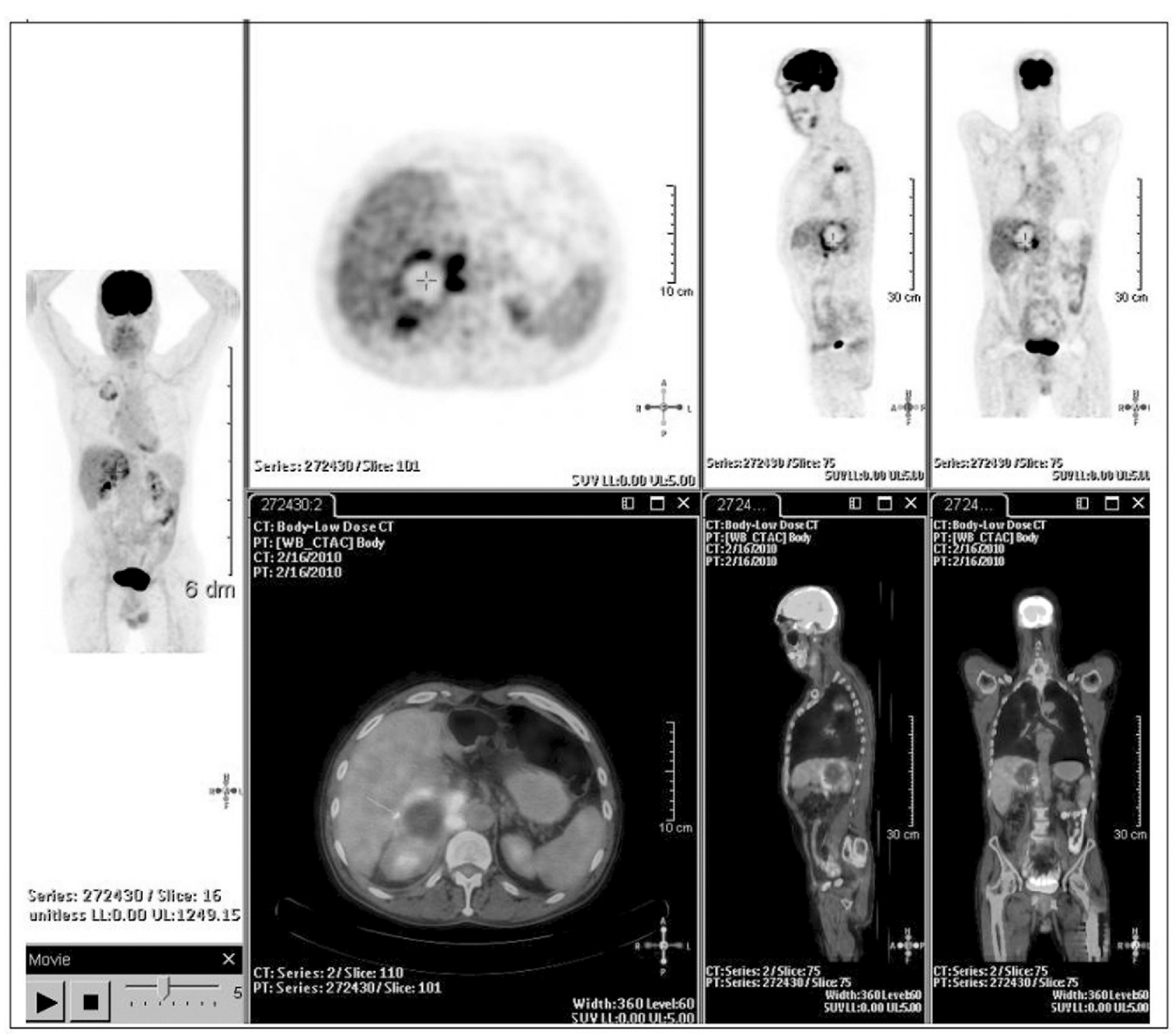

Fig. 7. PET/CT scan of the whole body displays a nodule lesion in the right suprarenal gland with high glucose metabolism in the borders SUV 4.3

(Fig.8). The result of the biopsy showed a metastasis from a non-small lung cancer.

- A - cells from adrenal cortex-loosely cohesive fragment of adrenocortical tissue, without significant nuclear pleomorphism and abundant granular cytoplasm with focal lipofuscin pigment.

- B - hypercelluar crowded fragment of malignant epithelium, prominent cellular pleomorphism and nuclear hyperchromasia.

- C, D, E - sharply outlined groups of malignant cells. No signs of granulomatous inflammation.

Figure B would suggest primary adenocortical carcinoma, but C, D, E favor metatstatic disease - non-small cell lung cancer (in the setting of clinical information).

\section{CONCLUSION}

Integrated PET/CT is better imaging technique than $\mathrm{CT}$ alone, PET alone and visual correlation of PET and CT. PET sets the gold standard in the evaluation of indeterminate solitary pulmonary nodule or mass, where PET has proven to be significantly more accurate than computer tomography in the distinction between benign and malignant lesions.

With integrated PET/CT, tumors can be better delineated from surrounding structures, such as chest wall, mediastinum or surrounding atelectasis.

$\mathrm{PET} / \mathrm{CT}$ increases the specificity and positive predictive value, owing to the combination of metabolic and anatomic information.

However, there are still many inderminate lesions that need hystopatological proof, and integrated PET/CT can be helpful in guiding these interventional procedures.

PET/CT is very helpful for the differential diagnosis between lung cancer and pulmonary tuberculosis. 

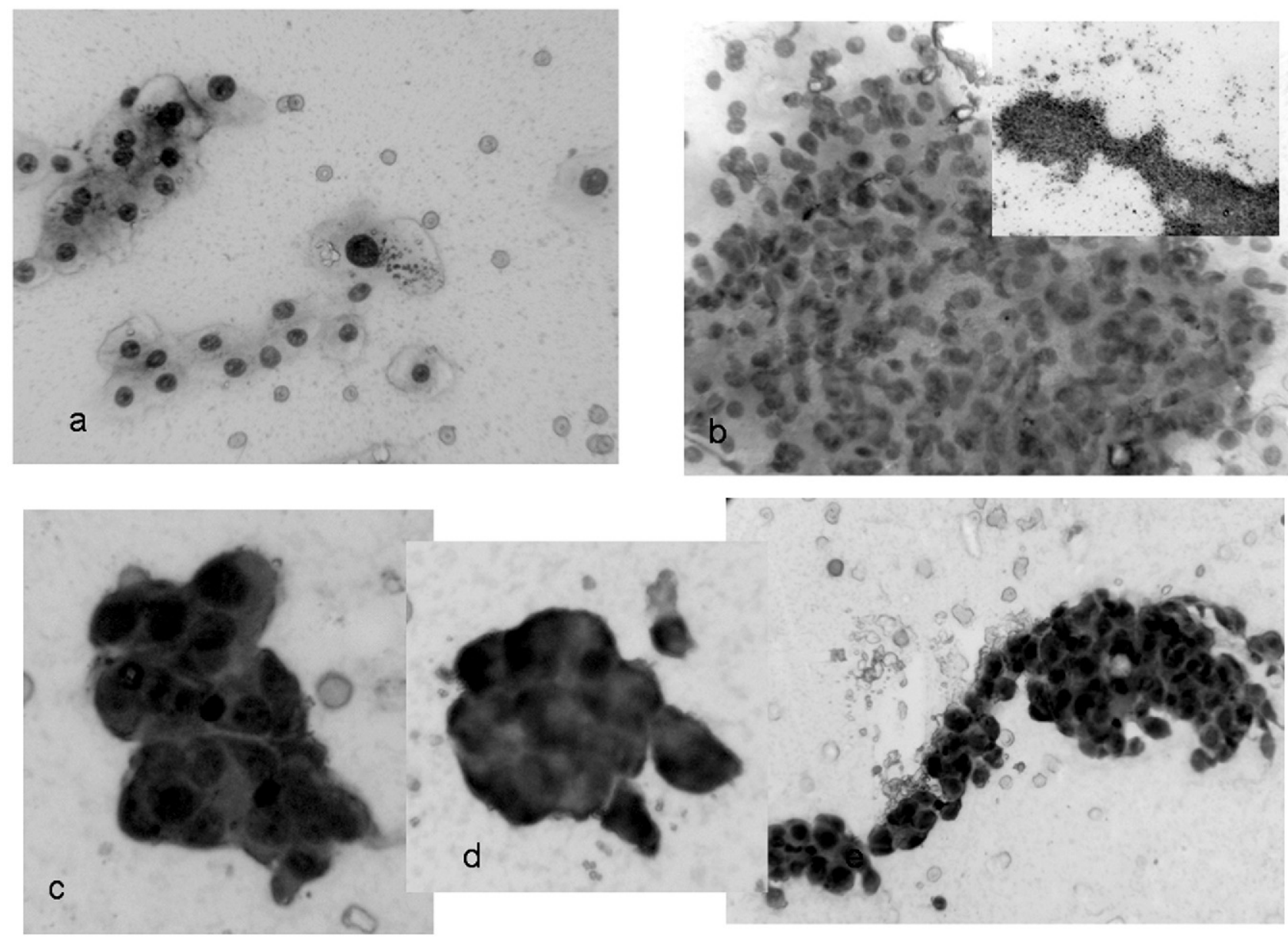

Fig. 8. FNAB-adrenal gland-Giemsa stain - $10 \times 20$ and $10 \times 40$

\section{REFERENCES}

1. Chaudhuri MR. Primary pulmonary cavitating carcinomas.Thorax. 1973; 28:354 - 366.

2. Chiu FT. Cavitation in lung cancers. Aust NZJ Med. 1975; 5; 523-530.

3. Dodd GD, Boyle JJ. Excavating pulmonary metastases. Am. J Reoentgenol Radium Ther Nuc Med. 1961; 85:277-293.

4. Felson B, Wolt JF. Some less familiar roentgen manifestations of carcinoma of the lung. Semin Roentgenol.1977;12:187 - 206.

5. Im JG, Itoh H, Shim YS, et al. Pulmonary tuberculosis: CT findings - early active disease and sequentialchange with antituberculous therapy. Radiology. 1993; 186: 653 - 660.

6. Miller RR, McGregor DH. Hemorrhage from carcinoma of the lung.1980;46:200-205.
7. Mouroux J, Padovani B, Elkaim D,

Richelme H. Shoud cavitated bronchopulmonary cancers be considered a separate entity? Ann Thorac Surg. 1996;61:530 - 532 .

8. Onn A, Choe DH, Herbst RS, et al. Tumor cavitation in stage I non-small cell lung cancer: epidermal growth factor receptor expression and prediction of poor outcome. Radiology.2005; 237:342 - 347.

9. Rodrigo T, Cayla JA, Garcia de Olalla P, et al. Characterics of tuberculosis patients who generate secondary cases. Int J Tuberc Lung Dis. 1997; 1: 352-357.

10. Swesen SJ, Payne WS, Viggiano RW, Pairolero PC, Trastec VF. An integrated approach to evaluation of the solitary pulmonary nodule. Mayo Clin Proc 1990; 65:173-186. 